\title{
Auditory Pathology in a Transgenic mtTFB1 Mouse Model of Mitochondrial Deafness
}

\author{
Sharen E. McKay, ${ }^{\dagger \dagger}$ Wayne Yan, ${ }^{\ddagger}$ Jessica Nouws, ${ }^{*}$ Maximilian J. Thormann, ${ }^{*}$ Nuno Raimundo, ${ }^{\S}$ Abdul Khan, ${ }^{*}$ \\ Joseph Santos-Sacchi, ${ }^{\ddagger \mathbb{T} \|}$ Lei Song, ${ }^{\ddagger}$ and Gerald S. Shadel ${ }^{* * * *}$
}

From the Departments of Pathology, * Surgery, ${ }^{\ddagger}$ Cellular and Molecular Physiology, ${ }^{\llbracket}$ Neurobiology, $"$ and Genetics, $* *$ Yale School of Medicine, New Haven, Connecticut; the Department of Psychology, ${ }^{\dagger}$ University of Bridgeport, Bridgeport, Connecticut; and the Institute of Cell Biology, ${ }^{\S}$ University Medical Center Göettingen, Göttingen, Germany

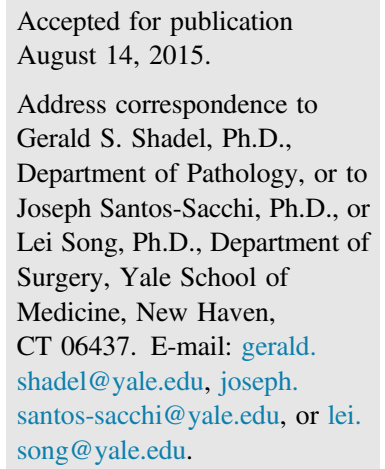

\begin{abstract}
The A1555G mutation in the 12S rRNA gene of human mitochondrial DNA causes maternally inherited, nonsyndromic deafness, an extreme case of tissue-specific mitochondrial pathology. A transgenic mouse strain that robustly overexpresses the mitochondrial $12 \mathrm{~S}$ ribosomal RNA methyltransferase TFB1M (Tg-mtTFB1 mice) exhibits progressive hearing loss that we proposed models aspects of A1555G-related pathology in humans. Although our previous studies of Tg-mtTFB1 mice implicated apoptosis in the spiral ganglion and stria vascularis because of mitochondrial reactive oxygen species-mediated activation of AMP kinase (AMPK) and the nuclear transcription factor E2F1, detailed auditory pathology was not delineated. Herein, we show that Tg-mTTFB1 mice have reduced endocochlear potential, indicative of significant stria vascularis dysfunction, but without obvious signs of strial atrophy. We also observed decreased auditory brainstem response peak 1 amplitude and prolonged wave I latency, consistent with apoptosis of spiral ganglion neurons. Although no major loss of hair cells was observed, there was a mild impairment of voltage-dependent electromotility of outer hair cells. On the basis of these results, we propose that these events conspire to produce the progressive hearing loss phenotype in Tg-mtTFB1 mice. Finally, genetically reducing AMPK $\alpha 1$ rescues hearing loss in Tg-mtTFB1 mice, confirming that aberrant up-regulation of AMPK signaling promotes the observed auditory pathology. The relevance of these findings to human $\mathrm{A} 1555 \mathrm{G}$ patients and the potential therapeutic value of reducing AMPK activity are discussed. (Am J Pathol 2015, 185: 3132-3140; http://dx.doi.org/10.1016/j.ajpath.2015.08.014)
\end{abstract}

Mitochondria are essential organelles that produce ATP via the process of oxidative phosphorylation, but are multifunctional, playing additional key roles in metabolism, as well as other cellular processes like apoptosis, inflammation, and signal transduction. ${ }^{1-4}$ Human mitochondria possess a 16,565-bp circular genome that is maternally inherited and present at hundreds to thousands of copies per cell in most tissues. ${ }^{5}$ In mammals, mitochondrial DNA (mtDNA) encodes 13 oxidative phosphorylation complex subunits and the two rRNAs and 22 tRNAs needed for translation of these by dedicated mitochondrial ribosomes. ${ }^{6}$ All other components of the estimated 1200-member mitochondrial proteome, including the remaining 70 to 75 oxidative phosphorylation subunits and all of the factors needed for mtDNA replication and expression, are encoded by nuclear genes and imported into the organelle. ${ }^{7}$ Thus, signaling pathways between mitochondria and the nucleus are required to coordinate the biogenesis, composition, and activity of mitochondria and to trigger homeostatic nuclear gene expression responses to mitochondrial dysfunction. These responses can be beneficial or harmful, depending on the precise cellular context and, to date, remain poorly understood.

Mitochondrial dysfunction causes human diseases, with an estimated occurrence of 1 in 5000 to 10,000 live births. $^{8-10}$ These can be inherited maternally, because of mutations in mtDNA, or in a Mendelian manner, because of mutations in

\footnotetext{
Supported by NIH grant AG047632 and a Yale Claude D. Pepper Older American Independence Center pilot grant (G.S.S.), an Ohse Research grant (L.S.), NIH National Institute on Deafness and Other Communication Disorders grants DC000273 and DC008130 (J.S.-S.), and European Molecular Biology Organisation (EMBO) postdoctoral fellowship EMBO ALTF 1328-2012 (J.N.).
}

Disclosures: None declared. 
nuclear genes encoding mitochondrial components. Because mtDNA is present in multiple copies per cell, and different organs vary in their energy requirements, mitochondrial diseases are complicated and heterogeneous, characterized by cell- and tissue-specific responses and pathology. ${ }^{2,8-10}$ An extreme example of tissue specificity is the A1555G mtDNA mutation that causes maternally inherited deafness. This mutation causes a progressive, nonsyndromic hearing loss that can vary from mild to profound and has a variable age of onset. ${ }^{11,12} \mathrm{~A} 1555 \mathrm{G}$ carriers can also be predisposed to aminoglycoside-induced deafness. ${ }^{13}$ Hearing loss induced by A1555G is incompletely penetrant, which has been attributed, at least in part, to multiple nuclear and mtDNA modifying loci. ${ }^{11,14,15}$ One such nuclear modifier is the TFB1M gene, which encodes an RNA methyltransferase that posttranscriptionally modifies the mtDNA-encoded $12 \mathrm{~S}$ rRNA in mitochondrial ribosomes. ${ }^{16}$

The A1555G mutation is located near a highly conserved stem loop in the 12S rRNA that contains adjacent adenines that are dimethylated by TFB1M. We showed previously that patient-derived A1555G cybrid cell lines exhibit increased stem-loop dimethylation and/or increased amounts of $12 \mathrm{~S}$ rRNA methylated at this site. ${ }^{17,18}$ These cells also exhibited enhanced apoptotic susceptibility in culture ${ }^{17,18}$ because of mitochondrial reactive oxygen species (ROS)-dependent activation of $5^{\prime}$-AMP-activated protein kinase (AMPK) that unmasks the pro-apoptotic function of the nuclear transcription factor E2F1. These phenotypes are recapitulated in cell lines that overexpress the TFB1M methyltransferase in the absence of the A1555G mutation. TFB1M binds to the mitochondrial RNA polymerase (POLRMT) in the context of mitochondrial ribosomes to promote proper $12 \mathrm{~S}$ rRNA methylation and ribosome biogenesis. ${ }^{19}$ Thus, disruption of these interactions in the mature ribosome or during ribosome assembly may cause unique perturbations in mitochondrial homeostasis and increased ROS production that promote deafness. How this unique mitochondrial perturbation leads to tissue-specific pathology remains unknown.

Consistent with the above pathogenic mechanism, we showed that transgenic mice that globally overexpress TFB1M (Tg-mtTFB1) exhibit premature hearing loss in an E2F1dependent manner. ${ }^{18}$ These mice have the increased $12 \mathrm{~S}$ rRNA methylation signature and AMPK up-regulation in multiple tissues, as well as increased caspase 3 staining and E2F1 up-regulation in two tissues in the inner ear, the stria vascularis and spiral ganglion neurons. This suggested that deafness in Tg-mtTFB1 mice is induced by a pro-apoptotic, mROS-AMPK-E2F1 pathway similar to the one we delineated in the $\mathrm{A} 1555 \mathrm{G}$ patient cybrids, making Tg-mtTFB 1 mice an indirect, yet potentially useful, animal model for maternally inherited deafness caused by the A1555G mutation. ${ }^{18}$ Herein, we have characterized the mechanism of hearing loss in Tg-mtTFB1 mice and addressed directly the involvement of AMPK signaling in the pathogenic response in vivo.

\section{Materials and Methods}

\section{Animals}

The original mixed C57BL/6J $\times \mathrm{SJL} / \mathrm{J}$ transgenic $\mathrm{Tg}-\mathrm{mtTFB} 1$ mice ${ }^{18}$ were extensively back-crossed to the C57BL/6J genetic background. F8 and F9 backcrossed animals were bred to generate the Tg-mtTFB1 and wild-type (WT) littermates used in electrophysiological and histological studies. F8 Tg-mtTFB1 mice were bred to C57BL/6J heterozygous knockouts of AMPK $\alpha 1^{+/-}\left(\right.$Prkaal $\left.^{+/-}\right)$obtained from Dr. Benoit Viollet (INSERM, Paris, France), ${ }^{20,21}$ and the resulting AMPK $\alpha 1^{+/-} /$Tg-mtTFB 1 mice were bred to AMPK $\alpha 1^{+/+}$for studies of AMPK knockdown.

\section{ABR Analysis}

Animals were anesthetized with either $48 \mathrm{mg} / \mathrm{kg}$ pentobarbital or $480 \mathrm{mg} / \mathrm{kg}$ chloral hydrate (i.p.), and all recordings were conducted in a sound-attenuating chamber (Industrial Acoustics Corp., Bronx, NY). A customized TDT3 system (Tucker-Davis Technologies, Inc., Alachua, FL) was used for auditory brainstem response (ABR) recordings. Subdermal needle electrodes (Rochester Electro-Medical, Inc., Lutz, FL) were positioned at the vertex (active, noninverting), the infra-auricular mastoid region (reference, inverting), and the neck region (ground). Differentially recorded scalp potentials were bandpass filtered between 0.05 and $3 \mathrm{kHz}$ over a 15-millisecond (ms) epoch. A total of 400 trials were averaged for each waveform for each stimulus condition.

Symmetrically shaped tone bursts were $3 \mathrm{~ms}$ long $(1 \mathrm{~ms}$ raised cosine on/off ramps and $1 \mathrm{~ms}$ plateau). All acoustic stimuli were delivered free field via a speaker (Tucker Davis Technologies, Inc., Part FF1 2021) positioned $10 \mathrm{~cm}$ from the vertex. Stimulus levels were calibrated using a 0.5 -in condenser microphone (model 4016; ACO Pacific, Belmont, CA) positioned at the approximate location of the animal's head during recording sessions and are reported in decibels sound pressure level $(\mathrm{dB}$ SPL; referenced to $20 \mu \mathrm{Pa}$ ). Stimuli of alternating polarity were delivered at a rate of approximately 20 per second.

Tone burst responses were collected in half octave steps ranging from 32 to $2.0 \mathrm{kHz}$. The effects of level were determined by decreasing stimulus intensity in 5-dB steps. A maximum stimulus level of $90 \mathrm{~dB}$ SPL was used first to avoid overstimulation. If the thresholds exceed $90 \mathrm{~dB}$ SPL, gain was adjusted to $40 \mathrm{~dB}$ to deliver a maximum stimulus level of $110 \mathrm{~dB}$ SPL. ABR thresholds were determined visually by noting the response waveforms exceeding a $1: 1$ signal to noise ratio. A two-way analysis of variance was used to determine the overall effect of genotype on ABR thresholds at different frequencies. The uncorrected Fisher's least significant difference test was used to make post hoc comparisons for determining statistical significance at each frequency between WT and Tg-mtTFB1 mice.

Latencies of the initial four ABR peaks were measured from animals aged 3 to 6 months or 9 to 12 months by 
setting time markers at maxima of the peaks and measuring the time from onset of stimulus to peaks. Measurements were made at 8 and $11 \mathrm{kHz}$ on traces with visible peaks. Latencies of peak I and central conduction time (peak I to IV) were used in the analysis. Amplitudes of peak I were assessed by taking the mean of the $\Delta \mathrm{V}$ of the upward and downward slopes of peak I.

\section{EP Measurements}

Tg-mtTFB1 and WT animals, aged 9 to 12 months, were anesthetized with sodium pentobarbital $(48 \mathrm{mg} / \mathrm{kg}$ body weight as initial dose and supplement as needed at $24 \mathrm{mg} /$ $\mathrm{kg}$ ). Animals were then placed onto a stereotaxic mouse head holder (MA-6N; Narishige, Tokyo, Japan) mounted onto a ball-and-socket stage and a magnetic base (M-RN56; Newport Corp.). The round window was exposed through a ventral approach by opening the bulla of the temporal bone. A sharp electrode (10 to $15 \mathrm{M} \Omega, 1 \mathrm{~B} 150 \mathrm{~F}-4$; World Precision Instruments, Sarasota, FL) with $3 \mathrm{~mol} / \mathrm{L}$ $\mathrm{KCl}$ pipette solution was mounted onto a micromanipulator with a pulse motor driving unit (PF5-1; Narishige). Electrodes were first placed at the round window, with visualization under a surgical microscope. An Axon 200A patch clamp amplifier was used for current clamp recording with an Axon Digidata 1321A and jClamp software version 22.8.4 (Scisoft, Inc., Ridgefield, CT). When the electrode was inserted into the scala tympani, voltage was balanced to $0 \mathrm{mV}$ and then the electrodes were advanced through the basilar membrane into the scala media to measure the endocochlear potential (EP). Pipettes were then withdrawn back to the scala tympani or advanced through the scala vestibuli for confirmation of EP. Data analyses were performed offline.

\section{NLC Measurements}

Whole cell patch clamp recordings were made from single isolated outer hair cells (OHCs) from the organ of Corti of TgmtTFB1 and WT mice. The temporal bones were excised, and the cochleae were dissected free. Enzyme treatment $(1 \mathrm{mg} / \mathrm{mL}$ dispase I, 10 to 12 minutes) preceded gentle trituration, and isolated $\mathrm{OHCs}$ were placed in a glass-bottom recording chamber. An E600-FN microscope (Nikon, Tokyo, Japan) with a $40 \times$ water immersion objective was used to observe cells during voltage clamp. Experiments were performed at room temperature.

The base high chloride ionic blocking solution contained (in $\mathrm{mmol} / \mathrm{L}$ ) the following: $\mathrm{NaCl}, 100$; TEA-Cl, 20; $\mathrm{CsCl}$, 20; $\mathrm{CoCl}_{2}, 2 ; \mathrm{MgCl}_{2}, 1 ; \mathrm{CaCl}_{2}, 1$; and HEPES, 10. Base intracellular solutions contained (in $\mathrm{mmol} / \mathrm{L}$ ) the following: $\mathrm{CsCl}, 140 ; \mathrm{MgCl}_{2}, 2$; HEPES, 10; and EGTA, 10. An Axon 200B amplifier was used for the whole-cell patch-clamping recording. Nonlinear capacitance (NLC) was measured using a continuous, high-resolution ( $2.56 \mathrm{~ms}$ sampling), two-sine stimulus protocol $(10-\mathrm{mV}$ peak at both 390.6 and $781.2 \mathrm{~Hz}$ ) superimposed onto the voltage ramp range from
-200 to $200 \mathrm{mV} .^{22,23}$ Capacitance data were fit to the first derivative of a two-state Boltzmann function.

$$
\begin{gathered}
C_{m}=Q_{\max } \frac{z e}{k T} \frac{b}{(1+b)^{2}}+C_{l i n} \\
\text { where } b=\exp \left(\frac{-z e\left(V_{m}-V_{p k C m}\right)}{k T}\right) .
\end{gathered}
$$

$Q_{\max }$ is the maximum nonlinear charge moved, $\mathrm{V}_{\mathrm{h}}$ is voltage at peak capacitance or equivalently, at half maximum charge transfer, $V_{m}$ is membrane potential, $z$ is valence, $C_{\text {lin }}$ is linear membrane capacitance, $e$ is electron charge, $k$ is Boltzmann constant, and $T$ is absolute temperature.

\section{Hair Cell Counts}

The temporal bones of 9- to 12-month-old WT ( $n=5$ ) were dissected, and cochlea removed and immersion fixed for 24 to 48 hours in $4 \%$ paraformaldehyde in phosphate-buffered saline (PBS). Bony capsules were partially dissected to enable fluid penetration, and the entire structure was then transferred to a blocking solution containing $10 \%$ normal goat serum and $0.1 \%$ Tween 20 in PBS. Mouse monoclonal Myo7a antibody (Developmental Hybridoma Studies Bank, University of Iowa, Iowa City, IA) was applied in blocking buffer at a 1:500 dilution overnight at $4^{\circ} \mathrm{C}$ and detected using an Alexa 546 donkey anti-mouse secondary antibody (Jackson Immunological, West Grove, PA). The bony capsule was then carefully removed, and the organ of Corti was detached from the modiolus as a single strip. Spiral ligaments were then dissected away to allow good exposure of the organ of Corti. The organ of Corti was then dissected and mounted. The apical region ranged from $57 \%$ to $64 \%$ from the base. This region has been described by Müller et $\mathrm{al}^{24}$ as corresponding to frequencies between 13.5 and $16 \mathrm{kHz}$, which encompasses the range of frequencies showing elevated ABR thresholds in the Tg-mtTFB1 mice. Myo7a-labeled inner hair cells (IHCs) and OHCs in this region were visualized with an IX-71 inverted fluorescence microscope (Olympus, Center Valley, PA), and images were collected and analyzed with a Spot camera (Diagnostic Instruments, Inc, Sterling Heights, MI). The number of cells was normalized and expressed as hair cells per $100 \mu \mathrm{m}$.

\section{Stria Vascularis Measurements}

Temporal bones were dissected and fixed in $4 \%$ paraformaldehyde in PBS at $4{ }^{\circ} \mathrm{C}$ for 24 to 48 hours, followed by decalcification in 10\% EDTA in PBS for 96 hours. The cochleae were bisected in the midmodiolar plane, and the two halves were embedded in paraffin, divided into sections, and stained with hematoxylin and eosin (Yale Pathology Developmental Histology Service, New Haven, CT). The width and thickness of intact, cross-sectioned striae were measured at multiple points at apical-basal locations ${ }^{25,26}$ in each animal using an Olympus microscope and Spot camera software version 5.1 for analysis. Strial width was measured as the 

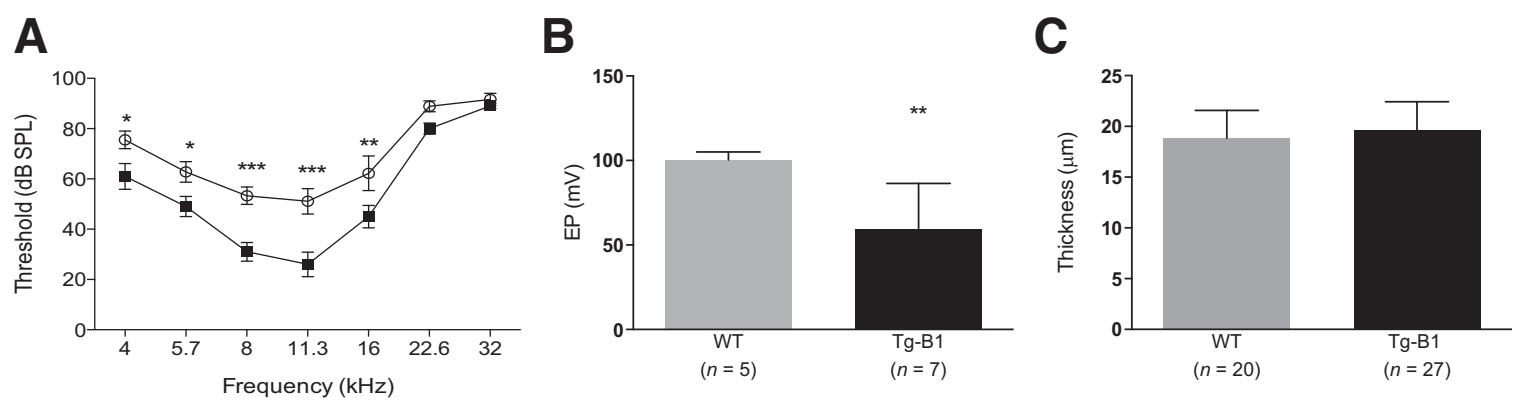

Figure 1 Hearing loss phenotype and stria vascularis dysfunction in a transgenic mouse strain that robustly overexpresses the mitochondrial 125 ribosomal RNA methyltransferase TFB1M (Tg-mtTFB1). A: Auditory brainstem response (ABR) results in wild-type (WT; closed squares) and Tg-mtTFB1 (Tg-B1; open circles) mice used in this study. Data were analyzed by two-way analysis of variance, and the main effect for genotype is $F_{(1,84)}=38, P<0.001$. Differences at each frequency were tested via post hoc Fisher's least significant difference tests. B: Direct endocochlear potential (EP) measurements of the mice in A. There is a significant EP reduction in Tg-mtTFB1 (Tg-B1, black bars) mice compared with WT (gray bars) controls [Student's $\mathrm{t}_{(10)}=3.3, * * P<0.01$ ] . C: Measurements of the thickness of the stria from hematoxylin and eosin-stained sections. No significant difference exists between WT and Tg-B1 mice $\left[\mathrm{t}_{(45)}=0.9\right] .{ }^{*} P<0.05,{ }^{*} P<0.01$, and ${ }^{* *} P<0.001 . \mathrm{dB}$ SPL, decibels sound pressure level.

length of a curved line between the two end points of the stria in cross section, one near the insertion of Reissner's membrane and the other near the spiral ligament. Thickness was measured by a straight line drawn through the midpoint of the section.

\section{Results}

\section{Hearing Loss-Related Pathology in the Stria Vascularis}

We previously characterized hearing loss in Tg-mtTFB 1 mice as elevated ABR thresholds that progressed with age, accompanied by increased caspase 3 staining in the stria vascularis and spiral ganglia and reduced numbers of spiral ganglion neurons. ${ }^{18}$ Because defects in the stria vascularis, a three-layered epithelial organ lining the scala media and responsible for maintaining the requisite elevated extracellular potassium necessary for hair cell function, can also result in hearing loss characterized by increased ABR thresholds, we measured the EP to assess strial function directly. After confirming that $\mathrm{ABR}$ thresholds were prematurely elevated in a cohort of Tg-mtTFB1 mice now extensively backcrossed to the C57BL/6J background (Figure 1A), we recorded EP in a group of WT and Tg-mtTFB1 animals aged 9 to 12 months. We observed a $40 \%$ reduction in EP in Tg-mtTFB1 mice $\left(\right.$ mean $\left._{\mathrm{Tg} \text {-mtTFB1 }}=60 \pm 10 \mathrm{mV}\right)$ compared with WT controls, which was near $100 \mathrm{mV}\left(\operatorname{mean}_{\mathrm{WT}}=100.2 \pm 2.1 \mathrm{mV}\right)$ (Figure 1B). Hearing loss accompanied by strial dysfunction in aging humans is often accompanied by atrophy of the epithelium. To assess atrophy, the width and thickness of the stria were measured at multiple sites in cochlear cross sections of nine Tg-mtTFB1 and six WT animals. The widths, indicators of the cochlear spiral location of the stria, ranged from 131 to 387 $\mu \mathrm{m}$ in WT and from 98 to $358 \mu \mathrm{m}$ in Tg-mtTFB1 animals. The thickness of the stria ranged from 13 to $22 \mu \mathrm{m}$ in the WT and from 14 to $28 \mu \mathrm{m}$ in the Tg-mtTFB1 animals. There was no significant correlation in either genotype between the width of the stria and the thickness (WT, $r^{2}=-0.0049$; Tg-mtTFB1, $\left.r^{2}=0.0077\right)$. Because there was no difference in the thickness as a function of apical-basal location, we combined all of the thickness measurements and compared the mean values by a $t$-test. There was no significant difference between WT and Tg-mtTFB1 mice (Figure 1C). These data suggest that the strial dysfunction in Tg-mtTFB1 mice is either distinct from presbycusis or we are sampling at a stage of progressive hearing loss that precedes gross strial atrophy.

To more precisely determine the source of auditory dysfunction, we analyzed details of the ABRs of WT and TgmtTFB1 mice with hearing loss. Mouse ABRs have five characteristic peaks, the first of which represents activity in nerve VIII. Changes in the latency or amplitude of peak I reflect dysfunctional cochlear and/or nerve VIII processing. By using two-way analysis of variance to assess the latency to peak I, we observed significant increases in 9- to 12-month-old Tg-mtTFB1 mice at both $8 \mathrm{kHz}\left[\mathrm{F}_{(1,109)}=34, P<0.001\right]$ (Figure $2 \mathrm{~A}$ ) and $11 \mathrm{kHz}\left[\mathrm{F}_{(1,127)}=28, P<0.001\right]$ (Figure 2B). When the same analysis was performed on 3- to 6-month-old animals, when only moderate ABR threshold shifts are observed in Tg-mtTFB1, the latencies were not significantly affected at $8 \mathrm{kHz}\left[\mathrm{F}_{(1,128)}=0.23\right]$ (Figure 2C) or $11 \mathrm{kHz}\left[\mathrm{F}_{(1,152)}=0.04\right]$ (Figure 2D). Thus, increased latency to peak I is only observed in older Tg-mtTFB1 animals with hearing loss. In contrast, central conduction time (elapsed time between peak I and peak IV), which reflects synaptic and nerve conduction timing after cochlear/nerve VIII processing, was unchanged in animals with severe hearing loss $\left[\mathrm{F}_{(1,104)}=1.2\right.$ and $\left.F_{(1,105)}=1.0\right]$ (Figure 2, E and F), respectively. These data suggest that hearing loss in Tg-mtTFB1 mice is induced by progressive pathological changes in the cochlea/nerve VIII, not central auditory system defects (ie, brainstem).

\section{Hearing Loss-Related Pathology in $\mathrm{OHCs}$}

To determine whether hair cells are directly affected, we dissected the organ of Corti from cochleae of 9-month-old WT and Tg-mtTFB1 mice and labeled IHCs and OHCs with an antibody for Myo7a. Despite considerable ABR threshold 

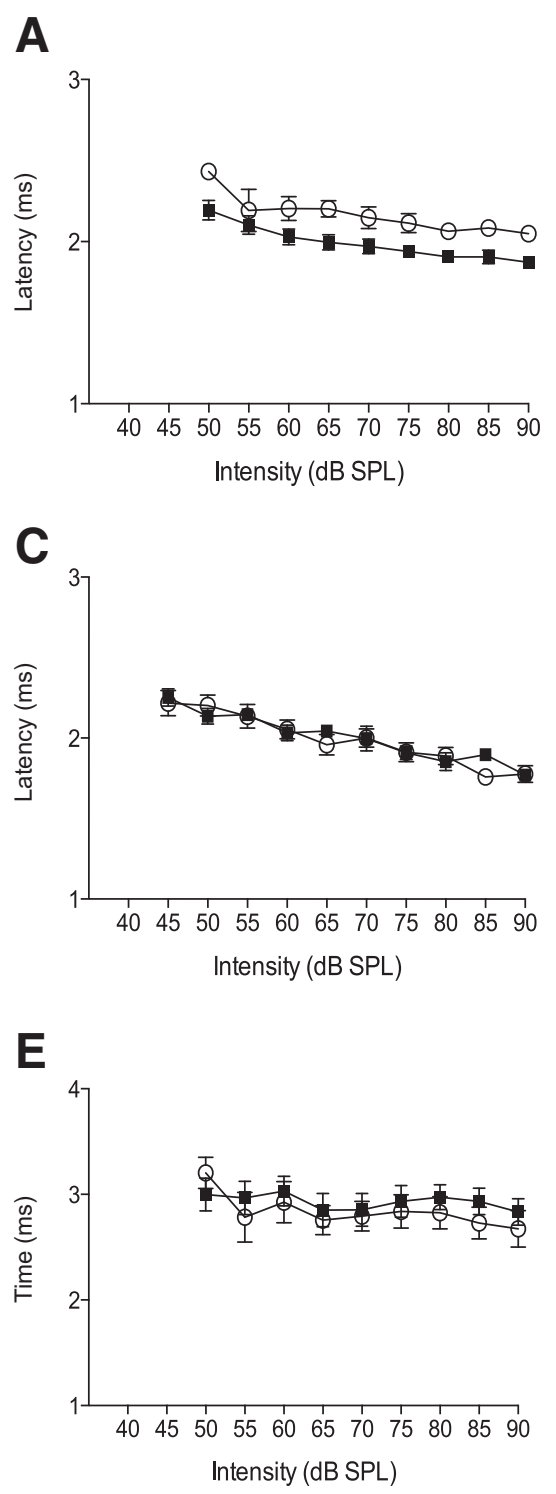

B
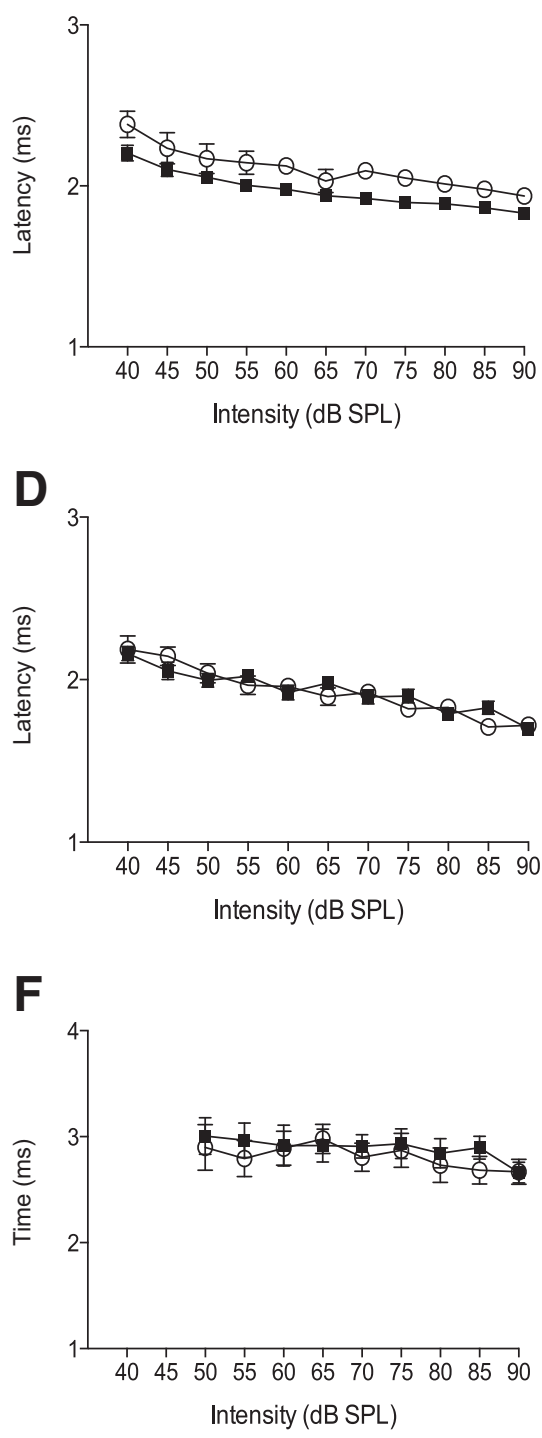

Figure 2 Changes in auditory brainstem response $(A B R)$ latencies demonstrate progressive cochlear damage underlying deafness in a transgenic mouse strain that robustly overexpresses the mitochondrial $12 S$ ribosomal RNA methyltransferase TFB1M (Tg-mtTFB1). The latency in milliseconds (ms) to ABR peak I is plotted as a function of the indicated suprathreshold decibels sound pressure level ( $d B \mathrm{SPL}$ ) for 9- to 12-month-old mice at $8 \mathrm{kHz}(\mathbf{A}), 9$ - to 12 -month-old mice at 11 $\mathrm{kHz}(\mathbf{B}), 3-$ to 6 -month-old mice at $8 \mathrm{kHz}(\mathbf{C})$, and 3- to 6-month-old mice at $11 \mathrm{kHz}$ (D). The central conduction time was measured as the time from ABR peak I to peak IV and is shown for 9- to 12month-old mice at $8 \mathrm{kHz}(\mathbf{E})$ and $11 \mathrm{kHz}(\mathbf{F})$. Closed squares indicate wild-type and open circles indicate Tg-mtTFB1 in all panels. changes at frequencies from 4 to $16 \mathrm{kHz}$ (Figure 1A), the hair cells in the apex of the cochlea, which detect frequencies in the range of hearing loss in Tg-mtTFB1 mice, ${ }^{24}$ are largely intact, as evidenced by quantifying Myo7a-labeled IHCs and OHCs in the most apical 2.5 to $3.0 \mathrm{~mm}$ of the organ of Corti (Figure 3A). Although there might be a downward trend in the number of hair cells in Tg-mtTFB 1 mice, a two-way analysis of variance shows that the counts do not differ significantly from WT $\left[\mathrm{F}_{(1,10)}=0.90\right]$.

Next, to determine whether OHC function was altered, NLC, the electrical correlate of electromotility, was measured in OHCs isolated from WT and Tg-mtTFB 1 mice between 9 and 12 months of age. NLC was measured by whole-cell patch clamp using symmetric chloride solutions $\left(140 \mathrm{mmol} / \mathrm{L} \mathrm{Cl}^{-}\right.$ intracellular and extracellular). OHCs from Tg-mtTFB1 mice exhibited a positive shift of nearly $20 \mathrm{mV}$ in the voltage at peak capacitance $\left(\mathrm{V}_{\mathrm{h}} ;\right.$ mean $_{\mathrm{WT}}=-94 \pm 2.6 \mathrm{mV}$; mean $_{\mathrm{Tg} \text {-mtTFB1 }}=-76 \pm 5 \mathrm{mV}$ ) (Figure 3B), suggesting that
TFB1M overexpression in the cochlea induces a long-term change in $\mathrm{OHC}$ voltage responsiveness.

\section{Functional Evidence for Spiral Ganglion Loss in Tg-mtTFB1 Mice}

To determine whether the approximately $20 \%$ spiral ganglion neuronal loss we observe in Tg-mtTFB1 mice ${ }^{18}$ has an effect on hearing, we analyzed ABR peak I amplitude, which reflects the number of spiral ganglion neurons recruited for the generation of ABR and would be reduced in animals with neuronal death in the spiral ganglion. Consistent with spiral ganglion loss, peak I amplitudes were significantly reduced in 9- to 12-month-old Tg-mtTFB1 animals $\left[\mathrm{F}_{(1,116)}=57, P<0.001\right]$ (Figure 3C). In addition, as stimulus levels increase, the characteristics of the curves in Tg-mtTFB1 mice diverge from those of WT, which show a steeper upward slope (recruitment) $>60 \mathrm{~dB}$ 

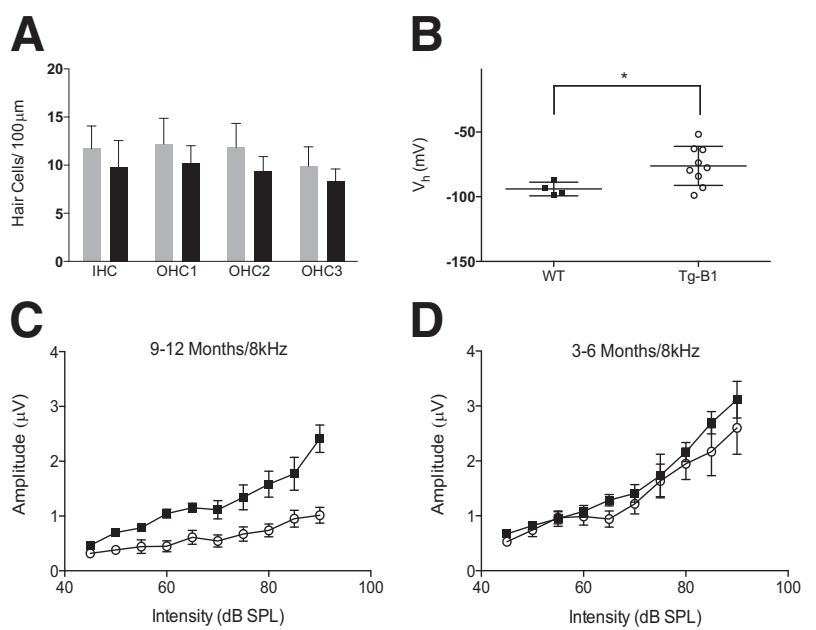

Figure 3 Outer hair cell dysfunction and reduced amplitude of auditory brainstem response (ABR) peak I in a transgenic mouse strain that robustly overexpresses the mitochondrial $12 \mathrm{~S}$ ribosomal RNA methyltransferase TFB1M (Tg-mtTFB1). A: Hair cell counts in wild-type (WT; gray bars) and TgmtTFB1 (Tg-B1; black bars) mice. Cells counted are as indicated: inner hair cells (IHCs), outer hair cell rows 1 (OHC1), 2 (OHC2), and 3 (OHC3). B: Nonlinear capacitance (NLC) recordings from $\mathrm{OHCs}$ isolated from WT (closed squares) and Tg-B1 (open circles) mice. The voltage at peak capacitance $\left(V_{h}\right)$ is plotted, and means were compared with a $t$-test. There is a statistically significant change in Tg-mtTFB1 $\left[\mathrm{t}_{(11)}=2.3\right]$. Other parameters of $\mathrm{NLC}$ were not different (means $\pm \mathrm{SD}$ ): maximum nonlinear charge moved $(\mathrm{pC}), \quad 0.54 \pm 0.11 \mathrm{WT} / 0.42 \pm 0.11 \mathrm{Tg}$-mtTFB1; $z, 0.76 \pm 0.05 \mathrm{WT} /$ $0.76 \pm 0.07 \mathrm{Tg}$-mtTFB1; linear membrane capacitance (pF), $4.93 \pm 0.47$ WT/4.44 $\pm 0.72 \mathrm{Tg}$-mtTFB1. The amplitude of ABR peak I was measured at $8 \mathrm{kHz}$ at the indicated intensities (decibels sound pressure level). Suprathreshold for 9- to 12-month-old mice (C) and 3- to 6-month-old mice (D). Suprathresholds at 9 to 12 months in Tg-mtTFB1 mice are significantly lower than WT controls by two-way analysis of variance. ${ }^{*} P<0.05$.

(Figure 3C). This suggests that Tg-mtTFB1 animals have an impaired cochlear amplifier, consistent with the findings of EP decrease (Figure 1B) and $\mathrm{OHC} \mathrm{V}_{\mathrm{h}}$ shift (Figure 3B). Younger animals with only moderately elevated ABR thresholds (3 to 6 months) had no significant change in the peak I amplitude $\left[\mathrm{F}_{(1,130)}=3.1, P>0.05\right]$ (Figure 3D), consistent with a progressive nature to the hearing loss in Tg-mtTFB1 mice.

\section{Rescue of Tg-mtTFB1 Hearing Loss by Genetically Reducing AMPK Signaling}

We proposed previously that mitochondrial ribosomal disruptions in Tg-mtTFB1 mice resulted in increased mitochondrial ROS-mediated activation of AMPK that engages the pro-apoptotic function of E2F1. ${ }^{18}$ In Tg-mtTFB1 mice, there is also increased phosphorylation of AMPK in the spiral ligament, a tissue that supports the K-recirculation function of the stria vascularis. ${ }^{18,27}$ If up-regulation of AMPK in the stria (or other cochlear cells) activates E2F1 to initiate apoptosis or other deleterious signaling events, then reducing AMPK activity in Tg-mtTFB1 mice would be predicted to rescue hearing loss. We bred Tg-mtTFB1 mice with heterozygous AMPK $\alpha 1$ subunit knockout mice ${ }^{20,21}\left(\mathrm{AMPK}^{+/-}\right)$to generate age-matched littermates of the following genotypes for ABR analysis: $\mathrm{AMPK}^{+/+} \mathrm{WT}$ (no transgene), $\mathrm{AMPK}^{+/+}$ Tg-mtTFB1 (with mtTFB1 transgene), $\mathrm{AMPK}^{+/-}$WT (no transgene), and $\mathrm{AMPK}^{+/-} \mathrm{Tg}-\mathrm{mtTFB} 1$ (with mtTFB1 transgene). At 9 to 12 months of age, we observed the typical increase in ABR threshold in Tg-mtTFB1 mice (Figure 4A). However, Tg-mtTFB1 mice with reduced AMPK signaling $\left(\mathrm{AMPK}^{+l-}\right.$ ) had an ABR threshold profile indistinguishable from WT littermate controls (Figure 4B). Thus, we conclude that reducing AMPK signaling has no effect on normal hearing at the ages tested, but rescues or delays premature hearing loss in Tg-mtTFB 1 mitochondrial deafness model mice.

\section{Discussion}

In this study, we have characterized the pathological mechanisms of hearing loss in Tg-mtTFB1 mice that we speculate models, to some degree, the pathology underlying maternally inherited deafness in humans because of the common A1555G mtDNA mutation. ${ }^{18}$ Our results demonstrate that hearing loss in Tg-mtTFB1 mice is because of cochlear dysfunction rather than a central processing defect in the brainstem (Figure 2). In the cochlea, we observe multiple defects, including signs of reduced spiral ganglion output (Figure 3C) and direct evidence for dysfunction in the stria vascularis (Figure 1B). We did not observe significant atrophy in the stria (at the light microscopic level), suggesting the defects involve intrinsic functioning of the cells in the tissue (eg, an inability to maintain the proper ionic milieu of the endolymph and EP within the scala media). The mitochondrial dysfunction in these cells may affect the functioning of the many ion pumps and transporters that require high and sustained amounts of ATP to operate. Alternatively, or in addition, these cells may be
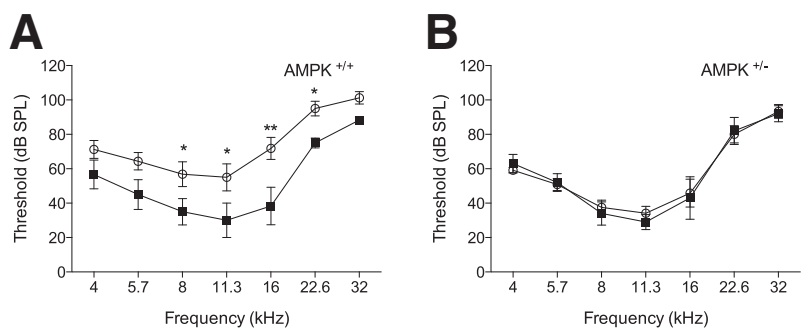

Figure 4 Reduced AMP kinase (AMPK) $\alpha 1$ signaling rescues hearing loss in a transgenic mouse strain that robustly overexpresses the mitochondrial $12 \mathrm{~S}$ ribosomal RNA methyltransferase TFB1M (Tg-mtTFB1). Auditory brainstem response thresholds of littermates of the indicated genotypes were tested at 9 to 12 months of age. A two-way analysis of variance demonstrates that the overall effect for genotype was statistically significant $\left[F_{(3,126)}=17\right.$, $\mathrm{P}<0.001$ ]. A: All animals are $\mathrm{AMPK}^{+/+}$(wild-type for AMPK $\alpha 1$ ) and either wild-type (non-transgenic, closed squares) or transgenic for mtTFB1 (TgmtTFB1, open circles). B: All animals are $\mathrm{AMPK}^{+/-}$(heterozygous for AMPK $\alpha 1$ ) and either wild-type (non-transgenic) or transgenic for mtTFB1 (Tg-mtTFB1). Results of post hoc Fisher's least significant difference tests comparing the genotypes at individual frequencies. ${ }^{*} P<0.05,{ }^{* \star} P<0.01$. db SPL, decibels sound pressure level. 
more prone to oxidation reduction perturbations and oxidative stress driven by mitochondrial ROS. Also, Schmitz et $\mathrm{al}^{26}$ observed reduced EP without morphological changes in the stria when hearing loss was induced with kanamycin and furosemide, which is similar to our results. We did not find evidence for major hair cell loss at ages when there is a clear ABR defect (Figure 3A), but there is some OHC dysfunction (Figure 3B). Interestingly, Niu et $\mathrm{al}^{28}$ showed that mice with accumulated mtDNA mutations have hearing loss with pathological features in the spiral ganglion and stria vascularis, but do not display major effects on hair cells, suggesting this may be a common profile of mitochondrial dysfunction in the inner ear. The loss of spiral ganglion neurons we reported previously ${ }^{18}$ is consistent with the reduced ABR peak I amplitude (Figure 3C) caused by fewer nerve VIII fibers contributing to the compound action potential. We propose that this loss of spiral ganglion neurons is because of apoptosis on the basis of the heightened caspase 3 staining in this region we reported previously. ${ }^{18}$ Furthermore, we propose that the defects we observe in the stria, spiral ganglion neurons, and OHCs conspire to produce the observed progressive hearing loss profile in Tg-mtTFB1 mice. Whether different pathogenic mechanisms downstream of mitochondrial ribosome disruptions in Tg-mtTFB1 mice occur independently in the three cell types involved or defects in one tissue occur as the result of a primary dysfunction in another cannot be determined from our results.

One interesting issue raised by our findings is the functional changes in the OHCs. Tg-mtTFB1 OHCs show a shift in NLC in vitro, suggesting that some chronic change in their responsiveness had occurred in vivo. Shifts in NLC are expected to have detrimental effects on cochlear amplification. ${ }^{29}$ It is possible that this shift is because of some intrinsic dysfunction. For example, the subsurface cisternae of the $\mathrm{OHC}$ that border the lateral membrane, which harbors the electromotile protein prestin, ${ }^{30,31}$ are lined by mitochondria, ${ }^{32}$ again suggesting that proper mitochondrial function may be necessary for normal $\mathrm{OHC}$ electromotility. Alternatively, stria dysfunction leading to altered composition of the endolymph (Figure 1B) may also produce some persistent cellular change in OHCs that we identified as a change in NLC. This could represent a new aspect of regulation of $\mathrm{OHC}$ activity.

Our previous study ${ }^{18}$ led to the formulation of a mitochondrial stress model that drives tissue-specific apoptotic signaling in the stria vascularis and spiral ganglion neurons. Specifically, altered mitochondrial ribosome activity and/or biogenesis because of premature or aberrant methylation of the $12 \mathrm{~S}$ rRNA by TFB1M, or direct inhibition of ribosome assembly on the basis of our recent report of physical and functional interactions between TFB1M and POLRMT in the ribosome, ${ }^{19}$ is proposed to lead to ROS-dependent activation of AMPK kinase and unveiling of the proapoptotic function of the nuclear transcription factor E2F1.$^{18}$ This model, based initially on in vitro results with cultured human A1555G cybrids, was partially verified in our previous study ${ }^{18}$ by showing the following: i) hearing loss in Tg-mtTFB1 mice did not occur when E2F1 levels were reduced genetically, ii) there is increased E2F1 expression and caspase 3 activation in the strial epithelium, and iii) there is activation of AMPK in the associated spiral ligament. Herein, we provide additional direct evidence that the mitochondrial ROS-AMPK-E2F1 pathogenic signaling pathway is operational in vivo by showing that hearing loss in Tg-mtTFB1 is rescued by genetically reducing AMPK signaling. The AMPK $\alpha 1$ form of the enzyme has been implicated in oxidative stress and ROS sensing, ${ }^{33-35}$ which is consistent with our results that hearing loss in Tg-mtTFB1 mice is rescued by inactivating one copy of the gene encoding AMPK $\alpha 1$, which would presumably reduce signaling from mitochondrial ROS. Finally, thioredoxin regulates the oxidation reduction sensitivity of AMPK. ${ }^{36}$ Thus, it will be interesting to determine whether this or other oxidation reduction-sensitive proteins are responsible for mediating mitochondrial ROS signals to AMPK in this pathogenic context and if antioxidants can also prove beneficial.

As a proposed animal model of the human deafnessassociated A1555G mtDNA mutation, a comparison of our results in Tg-mtTFB1 mice to the human condition and how this might inform prophylactic or therapeutic strategies is warranted. ${ }^{37,38}$ In human A1555G patients, hearing loss is considered to be a progressive, sensorineural deafness. Two important audiological characteristics of $\mathrm{A} 1555 \mathrm{G}$ patients compare favorably with Tg-mtTFB1. First, in A1555G carriers with normal hearing, there is a significant reduction in the distortion products of otoacoustic emissions ${ }^{39}$ (DPOAEs). Reduced DPOAE is interpreted as OHC dysfunction, yet these patients have normal ABRs, indicating hair cells must be largely intact. In this case, the reduced DPOAE might suggest that it is the stria that is not functioning properly, leading to some dysfunction of the OHCs, but not enough to affect the ABR at the time of testing. Our observations of NLC may be relevant, and it is possible that the magnitude of $\mathrm{OHC} \mathrm{V}_{\mathrm{h}}$ shift in these patients is not sufficient to influence threshold. These asymptomatic carriers of the A1555G mutation may later lose their hearing either as part of the normal progression of the disease or in response to aminoglycosides or other cochlear stressors (eg, intense noise) that we predict might activate the mitochondrial ROS-AMPK-E2F1 pathway. The second relevant comparison between humans and the mouse model involves the peak I latency. In both cases, peak I takes significantly longer to reach maximum amplitude, suggesting that cochlear processing and activation of spiral ganglion neurons are impaired. Interestingly, central conduction time is not affected in this model or in A1555G carriers, thus eliminating any brainstem abnormalities from the pathogenic process. ${ }^{39}$ One key difference between $\mathrm{Tg}$ mtTFB1 mice and A1555G is the involvement of hair cells in humans with profound hearing loss who have no 
measurable DPOAE, suggesting that they have no $\mathrm{OHCs}$ or they are seriously impaired. In contrast, Tg-mtTFB1 mice have intact $\mathrm{OHCs}$ and IHCs, as evidenced in this study both histologically and functionally. This may reflect a bona fide difference or a temporal difference in the progression of pathological events. Although future studies with older TgmtTFB1 mice might help clarify this, we acknowledge that studies of progressive hearing loss in the C57BL/6J background at older ages would become confounded by the agerelated hearing loss locus in these mice that, on its own, causes premature deafness. ${ }^{40,41}$ Studies of Tg-mtTFB1 in other genetic backgrounds, that vary dramatically in their propensity for hearing loss, ${ }^{42,43}$ should help clarify the precise degree that our Tg-mtTFB1 mice model human deafness caused by the $\mathrm{A} 1555 \mathrm{G}$ mutation.

Finally, it has recently been reported that lymphocytes and fibroblasts from A1555G patients do not exhibit increased 12S rRNA methylation like we observe in A1555G cybrid cells and Tg-mtTFB1 mice tissues. ${ }^{44}$ Whether this reflects a difference in how methylation is assayed by our two groups or if increased methylation only occurs in the inner ear (or just during stress) in humans remains to be determined. It is also formally possible that increased $12 \mathrm{~S}$ rRNA methylation of mature mitochondrial ribosomes is not the trigger of pathogenic responses in Tg-mtTFB1 mice or humans, as we proposed originally. For example, premature binding and/or methylation of $12 \mathrm{~S}$ rRNA by TFB1M (eg, during transcription or before RNA processing is complete) might disrupt ribosome biogenesis. Alternatively, we have recently shown that TFB1M is in a complex with POLRMT in ribosomes ${ }^{19}$; thus, overexpression of TFB1M and/or the A1555G mutation may disrupt the function of this complex in ribosomes to initiate the mROS-AMPK-E2F1 pathway and hearing loss. These mechanistic details will be important to decipher to better understand deafness caused by the A1555G mutation in humans and the degree to which it can be modeled by our Tg-mtTFB1 mice.

\section{Acknowledgments}

We thank Drs. Benoit Viollet and Lawrence Young for providing the AMP kinase knockout mice for this study, Dr. Peter Santi for sharing data on strial morphology, and Dr. Reuben Shaw for helpful advice on AMP kinase.

\section{References}

1. West AP, Shadel GS, Ghosh S: Mitochondria in innate immune responses. Nat Rev Immunol 2011, 11:389-402

2. Kasahara A, Scorrano L: Mitochondria: from cell death executioners to regulators of cell differentiation. Trends Cell Biol 2014, 24:761-770

3. Chandel NS: Mitochondria as signaling organelles. BMC Biol 2014, $12: 34$

4. Hill S, Van Remmen H: Mitochondrial stress signaling in longevity: a new role for mitochondrial function in aging. Redox Biol 2014, 2: 936-944
5. Shadel GS, Clayton DA: Mitochondrial DNA maintenance in vertebrates. Annu Rev Biochem 1997, 66:409-435

6. Shutt T, Shadel G: A compendium of human mitochondrial gene expression machinery with links to disease. Environ Mol Mutagen 2010, 51:360-379

7. Bestwick ML, Shadel GS: Accessorizing the human mitochondrial transcription machinery. Trends Biochem Sci 2013, 38:283-291

8. Wallace DC: A mitochondrial paradigm of metabolic and degenerative diseases, aging, and cancer: a dawn for evolutionary medicine. Annu Rev Genet 2005, 39:359-407

9. DiMauro S, Schon E: Mitochondrial respiratory-chain diseases. N Engl J Med 2003, 348:2656-2668

10. Vafai SB, Mootha VK: Mitochondrial disorders as windows into an ancient organelle. Nature 2012, 491:374-383

11. Guan MX, Fischel-Ghodsian N, Attardi G: Nuclear background determines biochemical phenotype in the deafness-associated mitochondrial 12S rRNA mutation. Hum Mol Genet 2001, 10:573-580

12. Prezant TR, Shohat M, Jaber L, Pressman S, Fischel-Ghodsian N: Biochemical characterization of a pedigree with mitochondrially inherited deafness. Am J Med Genet 1992, 44:465-472

13. Prezant TR, Agapian JV, Bohlman MC, Bu X, Oztas S, Qiu WQ, Arnos KS, Cortopassi GA, Jaber L, Rotter JI: Mitochondrial ribosomal RNA mutation associated with both antibiotic-induced and nonsyndromic deafness. Nat Genet 1993, 4:289-294

14. Zhao H, Li R, Wang Q, Yan Q, Deng J-H, Han D, Bai Y, Young W-Y, Guan M-X: Maternally inherited aminoglycoside-induced and nonsyndromic deafness is associated with the novel C1494T mutation in the mitochondrial $12 \mathrm{~S}$ rRNA gene in a large Chinese family. Am J Hum Genet 2004, 74:139-152

15. Dai P, Liu X, Han D, Qian Y, Huang D, Yuan H, Li W, Yu F, Zhang R, Lin H, He Y, Yu Y, Sun Q, Qin H, Li R, Zhang X, Kang D, Cao J, Young WY, Guan MX: Extremely low penetrance of deafness associated with the mitochondrial $12 \mathrm{~S}$ rRNA mutation in 16 Chinese families: implication for early detection and prevention of deafness. Biochem Biophys Res Commun 2006, 340:194-199

16. Bykhovskaya Y, Mengesha E, Wang D, Yang H, Estivill X, Shohat M, Fischel-Ghodsian N: Human mitochondrial transcription factor B1 as a modifier gene for hearing loss associated with the mitochondrial A1555G mutation. Mol Genet Metab 2004, 82:27-32

17. Cotney J, McKay SE, Shadel GS: Elucidation of separate, but collaborative functions of the rRNA methyltransferase-related human mitochondrial transcription factors $\mathrm{B} 1$ and $\mathrm{B} 2$ in mitochondrial biogenesis reveals new insight into maternally inherited deafness. Hum Mol Genet 2009, 18:2670-2682

18. Raimundo N, Song L, Shutt TE, McKay SE, Cotney J, Guan M-X, Gilliland TC, Hohuan D, Santos-Sacchi J, Shadel GS: Mitochondrial stress engages E2F1 apoptotic signaling to cause deafness. Cell 2012, $148: 716-726$

19. Surovtseva YV, Shadel GS: Transcription-independent role for human mitochondrial RNA polymerase in mitochondrial ribosome biogenesis. Nucleic Acids Res 2013, 41:2479-2488

20. Jørgensen SB, Wojtaszewski JFP, Viollet B, Andreelli F, Birk JB, Hellsten Y, Schjerling P, Vaulont S, Neufer PD, Richter EA, Pilegaard H: Effects of alpha-AMPK knockout on exercise-induced gene activation in mouse skeletal muscle. FASEB J 2005, 19:1146-1148

21. Faubert B, Boily G, Izreig S, Griss T, Samborska B, Dong Z, Dupuy F, Chambers C, Fuerth BJ, Viollet B, Mamer OA, Avizonis D, DeBerardinis RJ, Siegel PM, Jones RG: AMPK is a negative regulator of the Warburg effect and suppresses tumor growth in vivo. Cell Metab 2013, 17:113-124

22. Santos-Sacchi J, Kakehata S, Takahashi S: Effects of membrane potential on the voltage dependence of motility-related charge in outer hair cells of the guinea-pig. J Physiol 1998, 510(Pt 1): 225-235

23. Santos-Sacchi J: Determination of cell capacitance using the exact empirical solution of partial differential Y/partial differential $\mathrm{Cm}$ and its phase angle. Biophys J 2004, 87:714-727 
24. Müller M, von Hünerbein K, Hoidis S, Smolders JWT: A physiological place-frequency map of the cochlea in the CBA/J mouse. Hear Res 2005, 202:63-73

25. Santi PA, Muchow DC: Morphometry of the chinchilla organ of Corti and stria vascularis. J Histochem Cytochem 1979, 27:1539-1542

26. Schmitz HM, Johnson SB, Santi PA: Kanamycin-furosemide ototoxicity in the mouse cochlea: a 3-dimensional analysis. Otolaryngol Head Neck Surg 2014, 150:666-672

27. Wangemann P: Supporting sensory transduction: cochlear fluid homeostasis and the endocochlear potential. J Physiol 2006, 1:11-21

28. Niu X, Trifunovic A, Larsson N-G, Canlon B: Somatic mtDNA mutations cause progressive hearing loss in the mouse. Exp Cell Res 2007, 313:3924-3934

29. Santos-Sacchi J, Song L, Zheng J, Nuttall AL: Control of mammalian cochlear amplification by chloride anions. J Neurosci 2006, 26 : 3992-3998

30. Yu N, Zhu M-L, Zhao H-B: Prestin is expressed on the whole outer hair cell basolateral surface. Brain Res 2006, 1095:51-58

31. He DZZ, Jia S, Dallos P: Prestin and the dynamic stiffness of cochlear outer hair cells. J Neurosci 2003, 23:9089-9096

32. Spicer SS, Thomopoulos GN, Schulte BA: Cytologic evidence for mechanisms of $\mathrm{K}+$ transport and genesis of Hensen bodies and subsurface cisternae in outer hair cells. Anat Rec 1998, 251:97-113

33. Ju T-C, Chen H-M, Chen Y-C, Chang C-P, Chang C, Chern Y: AMPK- $\alpha 1$ functions downstream of oxidative stress to mediate neuronal atrophy in Huntington's disease. Biochim Biophys Acta 2014, 1842:1668-1680

34. Choi SL, Kim SJ, Lee KT, Kim J, Mu J, Birnbaum MJ, Soo Kim S, $\mathrm{Ha} \mathrm{J}$ : The regulation of AMP-activated protein kinase by $\mathrm{H}(2) \mathrm{O}(2)$. Biochem Biophys Res Commun 2001, 287:92-97
35. Wang S, Dale GL, Song P, Viollet B, Zou M-H: AMPKalpha1 deletion shortens erythrocyte life span in mice: role of oxidative stress. J Biol Chem 2010, 285:19976-19985

36. Shao D, Oka S-I, Liu T, Zhai P, Ago T, Sciarretta S, Li H, Sadoshima J: A redox-dependent mechanism for regulation of AMPK activation by Thioredoxin1 during energy starvation. Cell Metab 2014, 19:232-245

37. Tono T, Ushisako Y, Kiyomizu K, Usami S, Abe S, Shinkawa H, Komune S: Cochlear implantation in a patient with profound hearing loss with the A1555G mitochondrial mutation. Am J Otol 1998, 19:754-757

38. Sinnathuray AR, Raut V, Awa A, Magee A, Toner JG: A review of cochlear implantation in mitochondrial sensorineural hearing loss. Otol Neurotol 2003, 24:418-426

39. Bravo O, Ballana E, Estivill X: Cochlear alterations in deaf and unaffected subjects carrying the deafness-associated A1555G mutation in the mitochondrial 12S rRNA gene. Biochem Biophys Res Commun 2006, 344:511-516

40. Keithley EM, Canto C, Zheng QY, Fischel-Ghodsian N, Johnson KR: Agerelated hearing loss and the ahl locus in mice. Hear Res 2004, 188:21-28

41. Johnson KR, Zheng QY, Erway LC: A major gene affecting agerelated hearing loss is common to at least ten inbred strains of mice. Genomics 2000, 70:171-180

42. Johnson KR, Zheng QY, Noben-Trauth K: Strain background effects and genetic modifiers of hearing in mice. Brain Res 2006, 1091:79-88

43. Ohlemiller KK: Reduction in sharpness of frequency tuning but not endocochlear potential in aging and noise-exposed BALB/cJ mice. J Assoc Res Otolaryngol 2002, 3:444-456

44. O'Sullivan M, Rutland P, Lucas D, Ashton E, Hendricks S, Rahman S, Bitner-Glindzicz M: Mitochondrial m.1584A 12S m62A rRNA methylation in families with m.1555A $>\mathrm{G}$ associated hearing loss. Hum Mol Genet 2015, 24:1036-1044 